\title{
Atherosclerotic Coronary Artery Disease in Patients With Cardiometabolic Syndrome
}

\author{
Shin-ichiro Miura ${ }^{\mathrm{a}, \mathrm{b}}$, Yuhei Shiga ${ }^{\mathrm{a}}$, Amane Ike ${ }^{\mathrm{a}}$, Atsushi Iwata ${ }^{\mathrm{a}}$
}

\begin{abstract}
Major risk factors for cardiovascular disease (CVD) include aging, gender, smoking, family history and cardiometabolic syndrome. The relative residual risks for CVD after statin treatment for primary and secondary prevention have been reported by several large-scale randomized clinical trials. Statin treatment appears to prevent one-third of the onset and progression of CVD, but not the remaining two-thirds. There are three major problems regarding the residual risk of CVD: 1) Insufficient reduction of low-density lipoprotein cholesterol levels; 2) Low levels of high-density lipoprotein cholesterol and elevated triglyceride; and 3) Insufficient control of other risk factors (high blood pressure, obesity, metabolic syndrome, type 2 diabetes, etc.). Thus, a multifaceted preventive approach should be needed to prevent CVD after statin treatment.
\end{abstract}

Keywords: Cardiovascular disease; Cardiometabolic syndrome; Residual risks; Statin; Primary and secondary prevention

\section{Introduction}

Cardiometabolic syndrome is defined as metabolic dysfunction caused by a combination of insulin resistance, impaired glucose tolerance, dyslipidemia (DL), hypertension (HTN) and adiposity. Cardiometabolic syndrome is one of the risk factors for cardiovascular disease (CVD), along with aging, gender and family history. According to several large-scale randomized clinical trials, statin treatment for the primary and secondary prevention of CVD prevents one-third of the onset and progression of CVD, but not the remaining two-thirds [1]. The Treating to New Targets (TNT) Study indicated that the residual risk of CVD in statin-treated secondary prevention patients included both lipid-related and nonlipid factors, such as

Manuscript submitted March 11, 2019, accepted March 21, 2019

${ }^{a}$ Department of Cardiology, Fukuoka University School of Medicine, Fukuoka, Japan

${ }^{b}$ Corresponding Author: Shin-ichiro Miura, Department of Cardiology, Fukuoka University School of Medicine, 7-45-1 Nanakuma, Jonan-Ku, Fukuoka 814-0180, Japan.Email: miuras@cis.fukuoka-u.ac.jp

doi: https://doi.org/10.14740/cr857 increased body mass index, smoking, HTN, and diabetes mellitus (DM) [2].The relative residual risk of CVD is still a major problem after statin treatment. There are three major problems regarding the residual risk of CVD: 1) Insufficient reduction of low-density lipoprotein cholesterol (LDL-C) levels; 2) Low levels of high-density lipoprotein cholesterol (HDL-C) and elevated triglyceride (TG); and 3) Insufficient control of other risk factors (high blood pressure, obesity, metabolic syndrome, type 2 diabetes, etc.) (Table 1). Recently, we examined three Registries at Fukuoka University (FU) in Japan and identified risk factors for CVD for the primary and secondary prevention of atherosclerotic coronary artery disease (CAD): the Quantitative Coronary Angiography (FU-QCA) Registry [3-10], the Coronary Computed Tomography Angiography (FU-CCTA) Registry [11-21], and the Coronary Intravascular Ultrasound (FU-IVUS) Registry [22-27] (Table 2). Here, we review the problems that persist regarding the onset and progression of CVD independent of statin treatment based mainly on our data from these three Registries.

\section{Intensive Lipid-Lowering Therapy}

According to the Fourier study, evolocumab, which is a proprotein convertase subtilisin/kexin type 9 (PCSK9) inhibitor, dramatically reduced LDL-C levels (to $30 \mathrm{mg} / \mathrm{dL}$ ) compared to placebo (to $90 \mathrm{mg} / \mathrm{dL}$ ) [28]. Evolocumab also significantly suppressed the primary endpoint (CVD events, including CV death, myocardial infarction, stroke, hospitalization for unstable angina and coronary revascularization) compared to placebo. In addition, in patients with a previous acute coronary syndrome who were receiving high-intensity statin therapy (Odyssey Outcomes study), the risk of recurrent ischemic cardiovascular events was lower among patients who received alirocumab (PCSK9 inhibitor) than among those who received placebo [29].Thus, more intensive reduction of LDL-C after statin treatment is needed to prevent CVD.

\section{FU-QCA Registry}

The FU-QCA Registry [3-10] included data on 2,832 patients who underwent percutaneous coronary intervention (PCI): 1,158 of these patients allowed us to conduct a long-term (5 years) follow-up study [9]. QCA was quantified before and after PCI. The endpoint was major adverse cardiac events (major 
Table 1. Residual Risks of Cardiovascular Disease

Insufficient reduction of serum LDL-C levels

Low levels of serum HDL-C and elevated serum TG levels

Insufficient control of other risk factors (high blood pressure, obesity, metabolic syndrome, type 2 diabetes, etc.)

LDL-C: low-density lipoprotein cholesterol; HDL-C: high-density lipoprotein cholesterol; TG: triglyceride.

Table 2. Summary of Three FU Registries in Japan

\section{Registry name}

FU-QCA Registry: In female patients, HDL-C $<40 \mathrm{mg} / \mathrm{dL}$ and LDL-C $\geq 100 \mathrm{mg} / \mathrm{dL}$ were more strongly related to MACEs after adjusting for various factors including statin treatment. On the other hand, the combination was not related to MACEs in male patients.

FU-CCTA Registry: The number of significantly stenosed coronary vessels significantly increased as the number of metabolic factors increased. Lower levels of HDL-C may be most useful for predicting CAD independent of statin treatment.

FU-IVUS Registry: TG levels may be more important indicators of residual risk after statin treatment in women than in men.

\section{Chief researcher}

Dr. Ike

Dr. Shiga

Dr. Iwata

FU: Fukuoka University; QCA: Quantitative Coronary Angiography; LDL-C: low-density lipoprotein cholesterol; MACEs: major adverse cardiac events; CCTA: Coronary Computed Tomography Angiography; HDL-C: high-density lipoprotein cholesterol; CAD: coronary artery disease; IVUS: Coronary Intravascular Ultrasound; TG: triglyceride.

adverse cardiac events (MACEs): all-cause death, myocardial infarction, and target lesion revascularization). Lipid profile and medications were also evaluated. QCA was performed, and data on the minimum lumen diameter, reference diameters, \% diameter stenosis, and lesion length were obtained. Both men and women were divided into four groups according

a

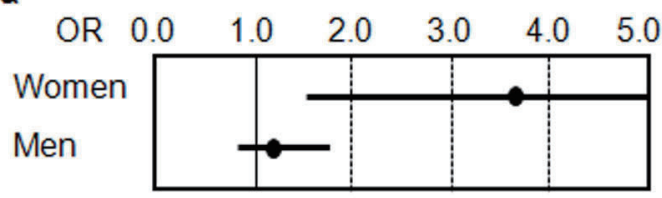

b
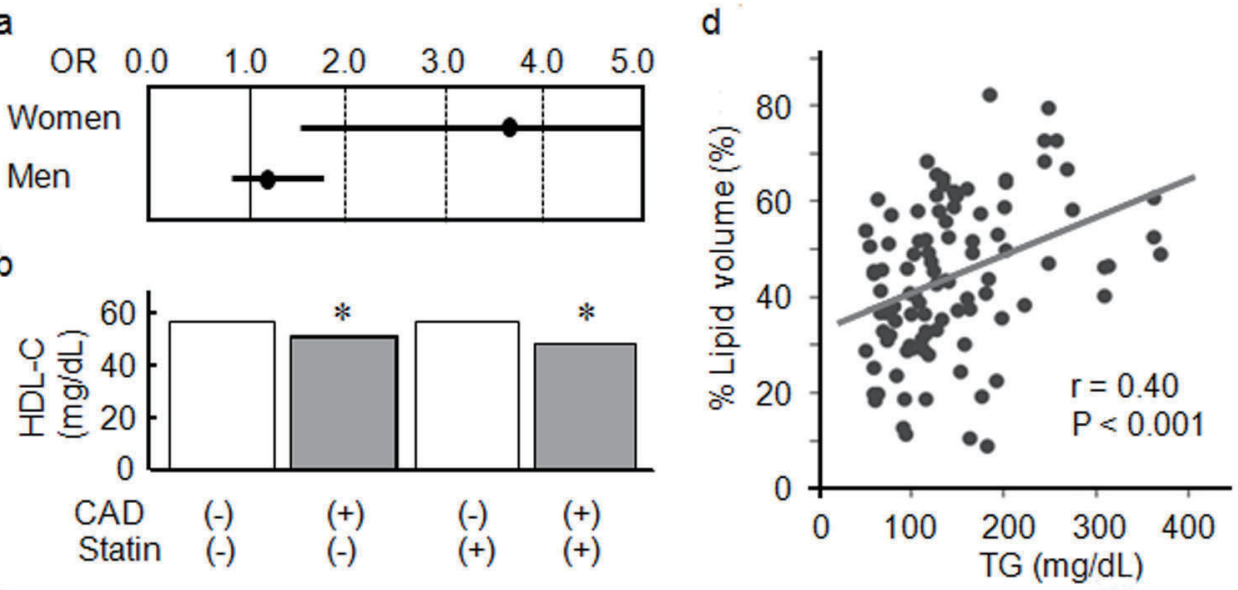

C

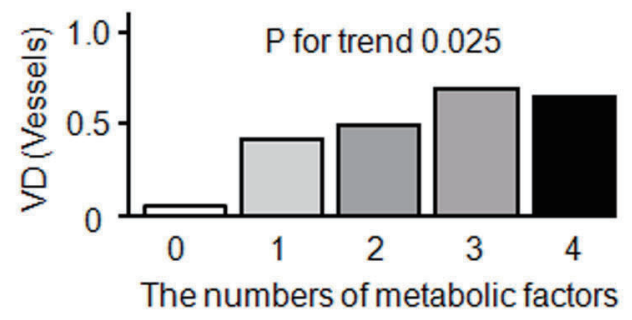

Figure 1. (a) Relationship between combination of HDL-C $<40 \mathrm{mg} / \mathrm{dL}$ and $\mathrm{LDL}-\mathrm{C} \geq 100 \mathrm{mg} / \mathrm{dL}$ and major adverse cardiac events after percutaneous coronary intervention. (b) Serum levels of HDL-C in patients with and without CAD in statin (-) and statin (+) groups. ${ }^{*} P<0.05$ versus CAD (-). (c) Association between the numbers of significant VD in coronary artery disease. (d) Association between serum levels of TG and \% lipid volume in women. HDL-C: high-density lipoprotein cholesterol; LDL-C: low-density lipoprotein cholesterol; CAD: coronary artery disease; OR: odds ratio; VD: stenosed vessels; TG: triglyceride. 
to the levels of HDL-C $40 \mathrm{mg} / \mathrm{dL}$ and LDL-C $100 \mathrm{mg} / \mathrm{dL}$. In female patients, HDL-C $<40 \mathrm{mg} / \mathrm{dL}$ and LDL-C $\geq 100 \mathrm{mg} / \mathrm{dL}$ were more strongly related to MACEs after adjusting for various factors including statin treatment (odds ratio (OR): 3.66 , coefficient interval (CI): $1.62-8.11, \mathrm{P}<0.001$ ) (Fig. 1a and Table 2). On the other hand, the combination of LDL-C with HDL-C was not related to MACEs in male patients (OR: 1.19, CI: $0.82-1.72, \mathrm{P}=0.36)$.

\section{FU-CCTA Registry}

About 1,000 patients who were clinically suspected to have CAD and who underwent CCTA were included in the FUCCTA Registry [11-21]. The number of significantly stenosed coronary vessels (VD, $\geq 50 \%$ coronary stenosis is diagnosed as CAD), and the Gensini score was quantified using CCTA. Lipid profile, adiponectin, pentraxin-3 and high-sensitivity C-reactive protein in blood were also measured. We evaluated coronary arteries using images showing volume-rendering, multi-planar reformation and cross-sectional views by CCTA. Shiga et al reported that serum levels of HDL-C in patients with CAD with and without statin therapy were significantly lower than those in patients without CAD with and without statin therapy (Fig. 1b) [14]. On the other hand, there were no differences in serum levels of LDL-C between patients with and without CAD. According to the results of a comparison of parameters with regard to the presence of $\mathrm{CAD}$, the presence of CAD was independently associated with both age (OR: 1.05, CI: $1.02-1.09$, $\mathrm{P}=0.003)$ and HDL-C (OR: 0.98, CI: $0.96-0.99, \mathrm{P}=0.04)$ in patients without statin treatment and with HDL-C (OR: 0.94, CI: $0.91-0.98, \mathrm{P}=0.0007$ ) in patients with statin treatment. Thus, HDL-C levels are most closely associated with the presence of CAD even after statin treatment. In particular, we need to perform CCTA in suspected CAD patients with lower HDL$\mathrm{C}$ levels under statin treatment (Table 2).

Mitsutake et al analyzed the association between the number of metabolic factors or plasma levels of adiponectin and the number of stenosed vessels in CAD [11]. Metabolic factors were defined as: 1) Visceral fat area $\geq 100 \mathrm{~cm}^{2}$; 2) Serum fasting glucose $\geq 110 \mathrm{mg} / \mathrm{dL}: 3$ ) Blood pressure $\geq 135 / 85 \mathrm{~mm}$ $\mathrm{Hg}$; 4) Serum $\mathrm{TG} \geq 150 \mathrm{mg} / \mathrm{dL}$; and 5) Serum HDL-C $<40$ $\mathrm{mg} / \mathrm{dL}$. In five groups classified according to the number of metabolic factors present, plasma adiponectin significantly decreased, whereas the number of stenosed vessels significantly increased as the number of metabolic factors increased (Fig. 1c and Table 2). According to a comparison of parameters based on the number of stenosed vessels by a multivariate logistic regression analysis [11], lower levels of HDL-C in addition to higher levels of LDL-C may be useful for predicting the severity of CAD independent of medications including statin treatment and other metabolic factors such as adiponectin and visceral fat area (Table 2).

\section{FU-IVUS Registry}

The FU-IVUS Registry [22-27] included about 400 CAD pa- tients who underwent PCI using integrated backscatter (IB)IVUS, and who were already receiving statin treatment. Lipid profiles including fasting serum TG levels were also measured. Gray-scale and IB-IVUS examinations were performed for a 10 -mm non-culprit segment of a coronary artery and the images were converted to plaque vulnerability. In both women and men, TG levels were not associated with coronary atheroma volume [26]. Interestingly, TG levels were significantly associated with lipid volume in women, but not in men, after statin treatment (Fig. 1d). Based on a multivariate stepwise regression analysis, TG $(\mathrm{P}<0.001), \mathrm{DM}(\mathrm{P}=0.04)$ and HTN $(\mathrm{P}$ $=0.04$ ) were independently associated with the percentage of lipid volume in the non-culprit segment of a coronary artery in women. On the other hand, only DM showed a similar association in men $(P=0.002)$. In summary, serum $T G$ levels were associated with lipid-rich coronary plaque in women, but not in men. The TG level may be a more important indicator of residual risk after statin treatment in women than in men (Table 2 ). There are gender differences in the associated factors.

\section{Conclusions}

Major residual risks for CVD include: 1) Insufficient reduction of LDL-C levels; 2) Low levels of HDL-C and elevated TG levels; and 3) Insufficient control of other risk factors. Although an increased number of metabolic factors are associated with more severe CAD, and lower HDL-C induces the onset and progression of $\mathrm{CAD}$, there may be gender differences. A multifaceted preventive approach should be needed to prevent CVD after statin treatment.

\section{Acknowledgments}

We thank all of the members of the Department of Cardiology, Fukuoka University, Japan. This work was presented as a Joint Session with Asia: Current and Future Perspectives in Cardiometabolic Syndrome at the second Asia-Pacific Cardiometabolic Syndrome Congress, February 22, 2019.

\section{Financial Disclosure}

None to declare.

\section{Conflict of Interest}

None.

\section{Author Contributions}

SM contributed to the conception and design of the work; YS, A Ike and A Iwata contributed to the acquisition, analysis, and interpretation of data for the work; YS, A Ike and A Iwata drafted the manuscript; SM critically revised the manuscript. 


\section{References}

1. Chapman MJ, Redfern JS, McGovern ME, Giral P. Niacin and fibrates in atherogenic dyslipidemia: pharmacotherapy to reduce cardiovascular risk. Pharmacol Ther. 2010;126(3):314-345.

2. Carrera E, Maeder-Ingvar M, Rossetti AO, Devuyst G, Bogousslavsky J, Lausanne Stroke R. Trends in risk factors, patterns and causes in hospitalized strokes over 25 years: The Lausanne Stroke Registry. Cerebrovasc Dis. 2007;24(1):97-103.

3. Ike A, Nishikawa H, Shirai K, Mori K, Kuwano T, Fukuda Y, Takamiya Y, et al. Impact of glycemic control on the clinical outcome in diabetic patients with percutaneous coronary intervention - from the FU-registry. Circ J. 2011;75(4):791-799.

4. Kuwano T, Miura S, Shirai K, Ike A, Mori K, Shimizu T, Zhang B, et al. Serum levels of bilirubin as an independent predictor of coronary in-stent restenosis: a new look at an old molecule. J Atheroscler Thromb. 2011;18(7):574583.

5. Sugihara M, Miura S, Nishikawa H, Ike A, Mori K, Iwata A, Kawamura A, et al. Characteristics of patients and types of lesions in patients with drug-eluting or bare-metal stent implantation in small coronary arteries: from the FU-Registry. J Cardiol. 2013;61(2):117-121.

6. Nagata I, Ike A, Nishikawa H, Zhang B, Sugihara M, Mori K, Iwata A, et al. Associations between lipid profiles and MACE in hemodialysis patients with percutaneous coronary intervention: from the FU-Registry. J Cardiol. 2015;65(2):105-111.

7. Ike A, Shirai K, Nishikawa H, Iwata A, Yahiro E, Sugihara M, Kawamura A, et al. Associations between different types of hypoglycemic agents and the clinical outcome of percutaneous coronary intervention in diabetic patientsFrom the FU-Registry. J Cardiol. 2015;65(5):390-396.

8. Gondo K, Ike A, Ogawa M, Shirai K, Sugihara M, Nishikawa $\mathrm{H}$, Iwata $\mathrm{A}$, et al. Is a bare-metal stent still useful for improving the outcome of percutaneous coronary intervention? From the FU-Registry. J Cardiol. 2017;69(4):652-659.

9. Matsuoka Y, Ike A, Ogawa M, Gondo K, Shirai K, Sugihara M, Nose D, et al. Sex difference between target levels of cholesterol-related parameters and post-PCI longterm clinical outcomes: From the FU-Registry. J Cardiol. 2018;71(3):259-267.

10. Shiiba M, Zhang B, Miura SI, Ike A, Nose D, Kuwano $\mathrm{T}$, Imaizumi S, et al. Association between discordance of LDL-C and non-HDL-C and clinical outcomes in patients with stent implantation: from the FU-Registry. Heart Vessels. 2018;33(2):102-112.

11. Mitsutake R, Miura S, Kawamura A, Saku K. Are metabolic factors associated with coronary artery stenosis on MDCT? Circ J. 2009;73(1):132-138.

12. Mitsutake R, Miura S, Zhang B, Saku K. HDL-associated factors provide additional prognostic information for coronary artery disease as determined by multi-detector row computed tomography. Int J Cardiol. 2010;143(1):72-78.
13. Mitsutake R, Miura S, Shiga Y, Uehara Y, Saku K. Association between hypertension and coronary artery disease as assessed by coronary computed tomography. J Clin Hypertens (Greenwich). 2011;13(3):198-204.

14. Shiga Y, Miura S, Mitsutake R, Kawamura A, Uehara Y, Saku K. Significance of serum high-density lipoprotein cholesterol levels for diagnosis of coronary stenosis as determined by MDCT in patients with suspected coronary artery disease. J Atheroscler Thromb. 2010;17(8):870878.

15. Shiga Y, Miura S, Mitsutake R, Yamagishi S, Saku K. Significance of plasma levels of pigment epitheliumderived factor as determined by multidetector row computed tomography in patients with mild chronic kidney disease and/or coronary artery disease. J Int Med Res. 2011;39(3):880-890.

16. Nakamura A, Miura S, Shiga Y, Norimatsu K, Miyase Y, Suematsu Y, Mitsutake R, et al. Is Pentraxin 3 a biomarker, a player, or both in the context of coronary atherosclerosis and metabolic factors? Heart Vessels. 2014;29:603610.

17. Norimatsu K, Miura S, Suematsu Y, Shiga Y, Miyase Y, Nakamura A, Yamada M, et al. Associations between glycated albumin or hemoglobin A1c and the presence of coronary artery disease. J Cardiol. 2015;65(6):487-493.

18. Yano M, Miura S, Shiga Y, Miyase Y, Suematsu Y, Norimatsu K, Nakamura A, et al. Association between smoking habits and severity of coronary stenosis as assessed by coronary computed tomography angiography. Heart Vessels. 2016;31(7):1061-1068.

19. Norimatsu K, Kuwano T, Miura SI, Shimizu T, Shiga Y, Suematsu Y, Miyase Y, et al. Significance of the percentage of cholesterol efflux capacity and total cholesterol efflux capacity in patients with or without coronary artery disease. Heart Vessels. 2017;32(1):30-38.

20. Ueda Y, Shiga Y, Idemoto Y, Tashiro K, Motozato K, Koyoshi R, Kuwano T, et al. Association between the presence or severity of coronary artery disease and pericardial fat, paracardial fat, epicardial fat, visceral fat, and subcutaneous fat as assessed by multi-detector row computed tomography. Int Heart J. 2018;59(4):695-704.

21. Nose D, Shiga Y, Ueda Y, Idemoto Y, Tashiro K, Suematsu Y, Kuwano T, et al. Association between plasma levels of PCSK9 and the presence of coronary artery disease in Japanese. Heart Vessels. 2019;34(1):19-28.

22. Iwata A, Miura S, Mori K, Kawamura A, Nishikawa H, Saku K. Associations between metabolic factors and coronary plaque growth or arterial remodeling as assessed by intravascular ultrasound in patients with stable angina. Hypertens Res. 2008;31(10):1879-1886.

23. Iwata A, Miura S, Morii J, Yamagishi S, Saku K. Association between plasma pigment epithelium-derived factor levels and tissue characteristics of coronary plaque using integrated backscatter intravascular ultrasound. Intern Med. 2011;50(18):1889-1894.

24. Iwata A, Miura S, Tanaka T, Ike A, Sugihara M, Nishikawa H, Kawamura A, et al. Plasma pentraxin-3 levels are associated with coronary plaque vulnerability and are decreased by statin. Coron Artery Dis. 2012;23(5):315- 
321.

25. Kato Y, Iwata A, Futami M, Yamashita M, Imaizumi S, Kuwano T, Ike A, et al. Impact of von Willebrand factor on coronary plaque burden in coronary artery disease patients treated with statins. Medicine (Baltimore). 2018;97(17):e0589.

26. Yamashita M, Iwata A, Kato Y, Futami M, Imaizumi S, Kuwano T, Ike A, et al. Impact of the triglyceride level on coronary plaque components in female patients with coronary artery disease treated with statins. Heart Vessels. 2018;33(10):1175-1184.

27. Shigemoto E, Iwata A, Futami M, Kato Y, Yamashita M,
Imaizumi S, Kuwano T, et al. Influence of chronic kidney disease on coronary plaque components in coronary artery disease patients with both diabetes mellitus and hypertension. Heart Vessels. 2019.

28. Sabatine MS, Giugliano RP, Keech AC, Honarpour N, Wiviott SD, Murphy SA, Kuder JF, et al. Evolocumab and clinical outcomes in patients with cardiovascular disease. N Engl J Med. 2017;376(18):1713-1722.

29. Schwartz GG, Steg PG, Szarek M, Bhatt DL, Bittner VA, Diaz R, Edelberg JM, et al. Alirocumab and cardiovascular outcomes after acute coronary syndrome. N Engl J Med. 2018;379(22):2097-2107. 